BULL. AUSTRAL. MATH. SOC.

VOL. $2(1970), 89-93$.

\title{
The weak closure of the set of singular elements in a Banach algebra
}

\section{J. D. Gray}

In this note it is proved that for a certain class of infinite dimensional Banach algebras the set of singular elements (the non-units) is dense in the weak topology.

It is well known and easily proven (Rickart, [2], p. 12), that in any (complex) Banach algebra $B$, with identity, the set $S$ of singular elements (the non-units) is closed in the norm topology. In some recent work of the author on a generalization of the operational calculus for Banach algebras it became important to know something of the topological nature of $S$ when $B$ is equipped with the weak topology. This topology has as a basis sets of the form

$$
\left\{\xi \in B:\left|x^{*}(x)-x^{*}(\xi)\right|<\varepsilon ; x^{*} \in A\right\}
$$

where $x \in B, \varepsilon>0$ and $A$ is a finite set in the dual space $B^{*}$ of continuous linear functionals on $B$. If $B$ (as a vector space) has finite dimension, the weak and the norm topology coincide, and so, in this case, $S$ is closed in the weak topology.

For a certain class of algebras we have a partial converse to this result.

THEOREM. Suppose $B$ is an infinite dimensional, semi-simple, commutative Banach algebra with identity, for which the Gelfand map is surjective. Then $S$ is weakly dense in $B$.

Proof. To see that this is a partial converse to the above statement

Received 15 September 1969. The author would like to thank Mr C.D. Cox for helpful discussions. 
we note that $S$ is always a proper subset of $B$, and so, if it is dense it is not closed. The Gelfand map is that well known homomorphism $B \rightarrow C(X)$ of $B$ into the Banach algebra $C(X)$ of all continuous, complex-valued functions on the compact Hausdorff space $X$ of maximal ideals of $B$. As $B$ is semi-simple, this homomorphism is injective, and thus, by assumption, bijective. By the open mapping theorem we conclude that it is a homeomorphism. Therefore, by Theorem V.3.15 of Dunford and Schwartz [1], it is a nomeomorphism when both $B$ and $C(X)$ have the weak topology. Thus it suffices to prove the theorem for the algebra $C(X)$. Now a function in $C(X)$ is singular if and only if it vanishes at some point of $X$. The problem is then: given $f \in C(X)$ (which we may assume not to be identically zero), show that every neighbourhood of $f$ in the weak topology contains a function which vanishes somewhere in $X$. It suffices therefore to exhibit a net $\left\{f_{\alpha}\right\}$ of singular elements with $\lim _{\alpha} f_{\alpha}=f$.

$f$ may be written in a unique way as $\phi+i \psi$, where $\phi, \psi: X \rightarrow R$ are continuous. Now, as $C(X)$ is infinite dimensional, $X$ is an infinite set, and as it is also compact, we conclude that there is a point $p \in X$ which is not isolated. Let $U$ be a neighbourhood basis for $p$ - indexed by some well-ordered set $\Gamma^{\prime}$, so $U=\left\{U_{\alpha}^{\prime}: \alpha \in \Gamma^{\prime}\right\}$. Choose an $\alpha_{0} \in \Gamma^{\prime}$ and define $U_{\alpha_{0}}=U^{\prime} \alpha_{0}$. If $\beta \geq \alpha_{0}$ and $U_{\beta}$ has been defined, define inductively $U_{\beta+}=U^{\prime}{ }_{\beta^{+}} \cap U_{\beta}$, where $\beta+$ is the least element of the set $\left\{\gamma \in \Gamma^{\prime}: \gamma>\beta\right\}$. Then the family $\left\{U_{\alpha}: \alpha \geq \alpha_{0}\right\}$ is also a neighbourhood basis of open sets for $p$. Furthermore, if $\beta>\alpha \geq \alpha_{0}$ we have $U_{\beta} \supset U_{\alpha}$, and each $U_{\alpha}$ contains a point other than $p$ (as $p$ is not isolated). Next, because $X$ is Hausdorff, we have $n_{\alpha \geq \alpha_{0}} U_{\alpha}=\{p\}$. For convenience we let $\Gamma$ be the directed set $\left\{\alpha \in \Gamma^{\prime}: \alpha \geq \alpha_{0}\right\}$. For each $\alpha \in \Gamma$ let $F_{\alpha}=X-U_{\alpha}$, so that $F_{\alpha}$ is closed. Also, let $\hat{F}_{\alpha}$ be the closed set $F_{\alpha} \cup\{p\}$. For $\alpha \in \Gamma$ we may, by construction, choose a point $p_{\alpha} \in U_{\alpha}$ in such a way that $p_{\alpha} \notin U_{\alpha+}$. $X$, being a compact Hausdorff space, is also normal, and hence, by Urysohn's Lemma, for each $\alpha \in \Gamma$, we may choose a continuous real-valued 
function $g_{\alpha}$ on $X$ so that $0 \leq g_{\alpha} \leq 1 ; g_{\alpha}\left(p_{\alpha}\right)=1$ and $g_{\alpha}$ vanishes on $\hat{F}_{\alpha}$. Now define $\phi_{\alpha}: X+R$ by

$$
\phi_{\alpha}(\lambda)=\left(1-g_{\alpha}(\lambda)\right) \phi(\lambda) ; \lambda \in X .
$$

Then $\phi_{\alpha}$ is continuous; $-\phi \leq \phi_{\alpha} \leq \phi ; \phi_{\alpha}\left(p_{\alpha}\right)=0$ and $\phi_{\alpha}\left|\hat{F}_{\alpha}=\phi\right| \hat{F}_{\alpha}$. In exactly the same manner we construct a continuous function $\psi_{\alpha}: X \rightarrow R$ with $-\psi \leq \psi_{\alpha} \leq \psi ; \psi_{\alpha}\left(p_{\alpha}\right)=0$ and $\psi_{\alpha}\left|\hat{F}_{\alpha}=\psi\right| \hat{F}_{\alpha}$. Write $f_{\alpha}=\phi_{\alpha}+i \psi_{\alpha}$ so that each $f_{\alpha}$ is a singular element of $c(X)$.

The net $\left\{f_{\alpha}: \alpha \in \Gamma\right\}$ will converge to $f$ in the weak topology if for each continuous linear functional $x^{*}$ on $C(X)$, and for each $\varepsilon>0$, there is an $\alpha_{1} \in \Gamma$ so that $\alpha>\alpha_{l}$ implies that

$$
\left|x^{*}(f)-x^{*}\left(f_{\alpha}\right)\right|<\varepsilon \text {. }
$$

The Riesz Representation Theorem ([1], Theorem IV.6.3) asserts the existence of an isometric isomorphism between $C(X)^{*}$ and the Banach space of regular, countably-additive, complex-valued measures on the Borel sets of $X$. Further, if $\mu$ is such a measure,

$$
x^{*}(g)=\int_{X} g d \mu
$$

for all $g \in C(X)$. Thus

$$
\left|x^{*}(f)-x^{*}\left(f_{\alpha}\right)\right| \leq\left|\int_{F_{\alpha}}\left(f-f_{\alpha}\right) d \mu\right|+\left|\int_{U_{\alpha}}\left(f-f_{\alpha}\right) d \mu\right| .
$$

However, for each $\alpha \in \Gamma$, the first factor above is identically zero as $f$ and $f_{\alpha}$ agree on $F_{\alpha}$. As for the second factor, it is

$$
\leq \int_{U_{\alpha}}\left|f-f_{\alpha}\right| \cdot d \| \mu||
$$

here $\|\mu\|$ represents the total variation of $\mu$, and, by Theorem III.5.12 of [1], illl is also a regular (positive) measure on the Borel sets of $X$. Now suppose that the $\|\mu\|$-measure of the point $p$ is zero. Then $\inf _{V}\|\mu\|(V)=0$ - the infimum being taken over all open sets $V$ 
containing $p$. Thus we can choose such an open set $V$ with $\|\mu\|(V)<\varepsilon / 2\|f\|$. But $\left\{U_{\alpha}\right\}$ is a basis for the open sets containing $p$, and as it is also decreasing, there is an $\alpha_{1} \in \Gamma$ such that, if $\alpha>\alpha_{1}$ we have $U_{\alpha} \subset V$ and so $\|\mu\|\left(U_{\alpha}\right)<\varepsilon / 2\|f\|$. Hence

$$
\int_{U_{\alpha}}\left|f-f_{\alpha}\right| \cdot d\|\mu\| \leq\left\|f-f_{\alpha}\right\| \int_{U_{\alpha}} d\|\mu\| \leq\left\|f-f_{\alpha}\right\| \cdot\|\mu\|\left(U_{\alpha}\right) \leq 2\|f\| \cdot\|\mu\|\left(U_{\alpha}\right)<\varepsilon
$$

provided $\alpha>\alpha_{1}$. Now suppose that $\{p\}$ does not have $\|\mu\|$-measure zero, then, without loss of generality, we may assume that $\|\mu\|(\{p\})=1$ and that $\int_{\{p\}}\left(f-f_{\alpha}\right) d \mu=0$ (remember that $\left.f(p)=f_{\alpha}(p)\right)$. Then

$$
\int_{U_{\alpha}}\left|f-f_{\alpha}\right| d\|\mu\|=\int_{U_{\alpha}-p}|f-f| d\|\mu\| \leq 2\|f\| \cdot\|\mu\|\left(U_{\alpha}-p\right) .
$$

By regularity we may choose an open set $V$ containing $p$ for which $\|\mu\|(V)<1+\varepsilon / 2\|f\|$ so that $\|\mu\|(V-p)<\varepsilon / 2\|f\|$. Arguing as before we find an $\alpha_{1}$ such that $\|\mu\|\left(U_{\alpha}-p\right)<\varepsilon / 2\|f\|$ provided $\alpha>\alpha_{1}$. This completes the proof of the theorem.

The proofs of the next results follow immediately from the theorem.

COROLLARY 1. Suppose $B$ is an infinite dimensionat, commutative, $B^{*}$-algebra with identity. Then $S$ is weakly dense in $B$.

COROLLARY 2. Let $X$ be an infinite compact Hausdorff space. Suppose $X$ contains a non-isolated point with a countable neighbourhood basis. Then $S$ is weakly sequentially dense in $C(X)$.

COROLLARY 3. Suppose the Banach algebra B satisfies the conditions of the theorem. Then, in the weak topology, the group of units of $B$ has empty interior.

Finally, let $B$ be the Banach algebra of bounded, complex-valued functions on a set $\Omega$. As mentioned in [2], p. 295, the group $G$ of units of $B$ is open and dense in the norm topology. However, in the weak topology, $G$ has empty interior. This follows from Corollary 3 and Theorem IV.6.18 of [1], according to which $B$ is (isometrically isomorphic to) an algebra $C(X)$, for a suitable compact Hausdorff space $X$. 


\section{References}

[.1] Nelson Dunford and Jacob T. Schwartz, Linear operators, Part I (Interscience Publishers, New York, London, 1958).

[2] Charles E. Rickart, General theory of Banach algebras, (Van Nostrand, Princeton, New Jersey, 1960).

University of New South Wales, Kensington, New South Wales. 